\title{
Articulación de perspectivas metodológicas: posibilidades del grupo de discusión para una sociedad reflexiva
}

\author{
Javier Callejo \\ U N ED . D epartamento de Sociología I \\ c/ O bispo Trejo, s/n. 28040 M adrid \\ jcallejo@uned.es
}

\section{Resumen}

La concreción de las distintas técnicas de investigación social tienen lugar en condiciones sociales determinadas. Por diversos autores, las sociedades contemporáneas son caracterizadas como reflexivas. ¿Q ué consecuencias tiene tal caracterización sobre la sociología empírica? Se ofrece una respuesta que tiene como referencia una práctica de investigación, el grupo de discusión, aplicada a campos que necesitan establecer unas mínimas líneas de consenso en medio del conflicto. Este tipo de aplicación cuestiona algunos criterios de clasificación e identificación de los distintos modos de hacer investigación social. Así, la barrera entre perspectiva estructural y perspectiva dialéctica, tomada de I báñez, desaparece. El artículo anal iza al gunas de las consecuencias prácticas sobre la técnica al situarse en el cruce de perspecivas: sobre la dinámica y el estilo de moderación.

Palabras clave: reflexividad, grupo de discusión, perspectiva estructural, perspectiva dialéctica, relación observador-observados.

\section{Abstract. M ethodological perspectives articulation: group discussion for a refl exive society}

In the concret, every social research instrument is applied in specific social conditions. Following sociologist as Beck, Giddens and others, contemporary societies are reflexive societies. H ow to take this in the empirical social research? This work offers a reply to the question using the focus group as reference, specially when the focus group is used in research? T his work offers a reply to the question using the focus group as reference, specially when the focus group is used in researchs with the aim to observe the posibilities of consensus. U sing the focus group in that way, we have found how some criteria to classify the ways of doing social research doesn't serve. So, the frontier between structural perspective and dialectic perspective, proposed by I bañez, di sappears. This essay analyse some of the practical consequences in the focus grop if we use it in the reflexive way. Special consideration to focus group dynamic and moderator role.

Key words: reflexivity, focus group, structural perspective, dialectic perspective, observerobserved relation. 


\section{Sumario}

\section{Introducción 5. El papel del moderador}

2. Las relaciones entre perspectivas 6. El reflexivo cierre dialéctico: metodológicas la percepción de la sociología por el grupo

3. Las bases de una sociología empírica reflexiva

4. Campos de actuación preferente de la 7. Conclusiones procedimentales Bibliografía articulación de perspectivas

La reflexividad es un instrumento para producir más ciencia, no para destruir la posibilidad de la ciencia. (Bourdieu, 1992: 67)

\section{Introducción}

El trabajo que aquí se presenta tiene por objetivo plantear: a) la actual validez de una práctica de investigación, el grupo de discusión, en unas condiciones social es concretas de aplicación; b) las relaciones entre perspectivas metodológicas. D esde el primer punto, es una reflexión sobre las modificaciones necesarias que ha de experimentar una práctica de investigación, como el grupo de discusión 1 , para adaptarse a un tipo de sociedad que, al menos, está dejando de ser la misma que le dio cuerpo práctico y teórico. Posibilidad de metamorfosis que parte de l báñez ${ }^{2}$, cuando afirma que: «en la misma técnica pueden estar incluidas varias perspectivas» (I báñez, 1990: 67), refiriéndose con el término pers pectivas a la división triádica que establece en la investigación social, según se busque la distribución de los fenómenos en una población (perspectiva distributiva), la estructura y las diferencias en su interior (perspectiva estructural) o sus posibilidades de transformación (perspectiva dialéctica).

La sociedad que sirvió de contexto a la producción teórica y práctica del grupo de discusión fue la sociedad de consumo de masas. Sin embargo, la sociedad actual empieza a encontrar problemas para poder seguir reconociéndose como tal ${ }^{3}$. U na sociedad que ya busca sus posibilidades de reproducción con cierta conciencia de impotencia, que demanda de manera manifiesta una

1. Utilizaré indistintamente los términos reunión de grupo y grupo de discusión, aun cuando puedan establecerse matices diferenciadores según el ámbito de referencia.

2. Jesús I báñez es, junto a Alfonso O rtí y Ángel de Lucas, la referencia básica en nuestro país al enfocar el grupo de discusión.

3. Véase, por ejemplo, al gunas de sus manifestaciones recientes en el diario Cinco días del 11 de noviembre de 1996. Procesos de globalización, dualización social, exclusión social en sociedades avanzadas ponen en jaque la equilibrada e integradora sociedad de consumo de masas (véase Callejo, 1995). En la medida que los sujetos han incorporado la sociedad de consumo, más se incorporan a ella como tales sujetos, quedando cada vez más de manifiesto, en los discursos coloquiales, que la relación con los objetos de consumo es una relación entre sujetos. 
investigación de lo posible, tras el general sentimiento de límite y agotamiento, que se encuentra en categorías como la de posmodernidad (Jameson, 1991). Pues bien, si algo parece definir la denominada perspectiva dialéctica de investigación social, siguiendo a I báñez, es la inclusión del campo de lo posible.

Introducirse en una sociología de las posibilidades es hacerlo en las posibilidades de la sociología. Sus posibilidades en un contexto que, al menos, cabe calificar de problemático. En estos casos: «cuando investigamos desde un contexto problemático, debemos hacer una articulación más o menos compleja de perspectivas y técnicas» (I báñez, 1990: 66).

\section{Las relaciones entre perspectivas metodológicas}

Siguiendo a I báñez (1985: 203 s.), las tres perspectivas metodológicas (distributiva, estructural y dialéctica) integran un mismo eje horizontal, que se cruza con un eje vertical formado por los niveles de la investigación (epistemológico, metodológico y tecnológico) que el autor toma de Bourdieu. En principio, cada una de las perspectivas es una particularidad en el nivel metodológico. Ahora bien, si la imagen de un eje genera la presencia de un gradiente limitado por los polos de lo puramente distributivo y lo puramente dialéctico, no están claras las hipotéticas áreas de encuentro entre dos perspectivas en el espacio generado.

Las tres perspectivas, a las que frecuentemente I báñez $(1985,1990)$ asimila con los niveles de la investigación, no están cerradas entre sí: «los tres niveles constituyen una unidad abierta que se está siempre reconstruyendo y desconstruyendo y que se expresa, integrando continuidades y discontinuidades» (Ibáñez, 1985: 204). N o obstante, el propio pliegue de un eje sobre otro que los aúna como niveles, y el hecho de que los niveles superiores tengan por objeto la negatividad de los niveles inferiores (lo que no pueden ser, lo que no puede ser dicho y no puede ser sabido desde el nivel inferior), hace ver que los caminos entre una perspectiva y otra no sean fácilmente franqueables; pues, del superior se puede acceder a los inferiores; pero no a la inversa. Un camino franqueable que, sin embargo, si se incorpora la dimensión pragmática (la acción) ocurre que: «si observamos un dispositivo de acción con un dispositivo de investigación de nivel inferior, lo destruimos al observarlo» (I báñez, 1990: 57). H ay un camino fácil para la destrucción y un camino difícil para la construcción. Volviendo a separar los dos ejes - niveles de observación, por un lado, perspectivas, por otro- cabe mayor posibilidad de flujo entre una perspectiva y otra, de manera que en el nivel tecnológico, en la misma técnica, como se veía antes, pueden estar incluidas varias perspectivas.

Las dos áreas de encuentro en el eje de perspectivas metodológicas son:

a) Área distributiva/estructural, que parece concretar los intentos de articulación de lo que habitualmente se clasifica como cualitativo y cuantitativo. En este aspecto, se ha pasado de la guerra entre cuantitativistas y cualitativistas a una especie de paz que, sin embargo, apenas ha superado la frontera 
entre una y otra forma de investigación. El análisis de redes parece ocupar actual mente el centro de este área entre perspectivas, aun cuando muchas de las esperanzas que este tipo de acercamiento a la realidad social originó hace más de treinta años 4 todavía están por desarrollarse 5 . N o obstante, con el uso de este tipo de análisis y partiendo de varios niveles de investigación, se ha posibilitado la actuación conjunta de técnicas cuantitativas (encuesta) y cualitativas (entrevistas abiertas).

Salvo en el caso de la potencialidad del análisis de redes, se trata de un área que vive entre las propuestas de aplicaciones complementarias (Alvira, 1983) o conjuntas (Conde, 1987, 1990), señalando en el último caso el carácter estructural de al gunos anál isis multivariables que se pueden practicar sobre datos producidos a partir de una encuesta. La propuesta de aplicación complementaria parte de la idea de que lo cualitativo y lo cuantitativo corresponden a lógicas distintas capaces de abordar, separadamente, aspectos asimismo distintos de la realidad social: dos perspectivas, como dos ojos, ven más que uno. La propuesta de articulación parte de no confundir la aritmética y los números con la matemática, incluyendo en ésta el orden topológico: ver lo cualitativo en lo cuantitativo es su principio. La primera opta por el diálogo entre perspectivas desde la asunción de que no es traducible una en la otra; la segunda apuesta por las posibilidades de una especie de código común, que posibilita la traducción de una en otra, a través de un esfuerzo de formalización.

b) El área de encuentro de perspectivas estructural/dialéctica ha sido apenas abordado, pues, entre otras dificultades, ha chocado con la de extraer del nivel micro de la organización o institución la aplicación de la perspectiva dialéctica. D e hecho, en el propio I báñez la perspectiva dialéctica se configura como un fantasma «más allá de la sociología» con escasa concreción, seguramente porque su concreción es del orden de la acción y no de las prescripciones metodológicas o tecnológicas. Incluso surgen dudas sobre si un conjunto difuso de actuaciones sociológicas realizadas bajo la categoría de investigación-participación-acción cabe incluirlo como perspectiva metodológica dialéctica cuando la descripción de experiencias toma la característica de una prescripción para «seguidores».

M e centraré en la segunda área de encuentro de perspectivas metodológicas, estructural/dialéctica, a partir de una práctica de investigación, la

4. D esde la sociología, cabe situar como trabajos seminales relevantes en el análisis de redes el de Coleman (1958) y Granovetter $(1973,1976)$, en la versión cuantitativa, y el de Bott (1971), en la versión cualitativa.

5. El análisis de redes ha recobrado importancia en su aplicación a las poblaciones escondidas (hidden populations), especialmente en el estudio de consumo de droga y las prácticas de riesgo de contagio del sida. Como ejemplos de este tipo de estudios, véase Spreen y Zwaagstra (1994), Sudman (1986), Spreen (1994), Watters y Biernacki (1989). Para el análisis de redes, véase, en castellano, las introducciones de Rodríguez (1995) y Lozares (1996). 
reunión de grupo. Como ocurre en el área interperspectiva distributiva/estructural del eje, el reto está en observar las capacidades de una técnica que se suele usar en un nivel de menor potencia (lo distributivo con respecto a lo estructural, lo estructural con respecto a lo dialéctico) para pasar a ser usada en un nivel de mayor potencia6.

Como toda perspectiva, la metodológica implica un espacio, una conciencia de posición con respecto al campo de observación. Una posición que enmarca lo observable - lo que se ha de recoger de la realidad social - para señalar posibilidades de acción en la investigación y en la sociedad. M etáfora de la espacialidad que indica más la estática que la dinámica, más la frontera como cierre que como puerta de paso. Una frontera que I báñez deja entreabierta para el caso de los grupos de discusión: «Es una frontera casi perfectamente continente: impide que la acción del grupo se propague a la sociedad global. Es una acción limitada y controlable (la frontera espacial del grupo está doblada por una frontera temporal, que se ciñe al tiempo de la discusión). Aunque no es imposible que el grupo permanezca, es poco probable» (I báñez, 1990: 63). La propia posibilidad de que el grupo pueda devenir grupo-sujeto, que permanezca y directamente actúe sobre la realidad social, abre el grupo a algunos rasgos de la perspectiva dialéctica, saliendo de su lugar como dispositivo de circulación de discursos y accediendo a las posibilidades de la acción y la transformación, aun cuando sea sólo como simulacro y dentro del control metodológico y, por lo tanto, subordinado a los intereses del macroconjunto, manteniendo los términos de Ibáñez. La posibilidad imaginaria de permanencia del grupo, de romper la barrera experimental de un lugar y un momento, permite abrir las fronteras entre las perspectivas estructural y dialéctica, de manera que pueden incrustrarse en el discurso del grupo las alternativas a la situación dominante, cuando el contexto es problemático, o la observación de la vinculación con los discursos. D e hecho, aun cuando sea sólo desde el punto de vista táctico, en contextos problemáticos: «debemos hacer una articulación más o menos compleja de perspectivas y técnicas» (I báñez, 1990: 66).

\section{Las bases de una sociología empírica reflexiva}

La necesidad de articular las perspectivas metodológicas estructural y dialéctica parte de: a) los cambios producidos en las sociedades occidentales avan-

6. Aun cuando, en el mejor de los casos, pudiera considerarse «matar moscas a cañonazos» cabe utilizar una técnica habitualmente ubicada en un nivel con mayor intensidad (menor extensión) en un nivel de mayor extensión (menor intensidad). Por ejemplo, se pueden diseñar múltiples reuniones de grupo, pasar a sus participantes un cuestionario y centrar el análisis en las respuestas a este cuestionario. I gualmente, se puede convocar asambleas en varias organizaciones - centros de trabajo, por ejemplo- y tomarlas y analizarlas como distintos grupos de discusión establecidos bajo el criterio de homogeneidad de trabajar en un mismo lugar. 
zadas, en las que la modernidad parece entrar por derroteros distintos a los que la caracterizaron durante el final del siglo XIX y la mayor parte del siglo XX, especialmente las identidades sociales aparecen como procesos en construcción por parte de los sujetos (nación, género, edad, subcultura, estilo de vida, etc. ); b) ciertos objetivos de la investigación social, que hacen más hincapié en la dimensión política de la misma, introduciéndose, de manera directa y manifiesta, en los conflictos, aun cuando tal introducción está siempre presente cuando se realiza investigación social; c) el papel protagonista de las instituciones públicas como demandantes, como «clientes», de investigación, a partir del objetivo de generar consensos. Como puede apreciarse, los puntos b y c están estrechamente conectados.

Los cambios sociales inciden en la forma de observarse la sociedad7. Se parte de la plausible hipótesis de que las transformaciones de la sociedad exigen transformaciones en las maneras de acercarse a ella para observarla y establecer la reflexión sobre la misma; pero, a su vez, tales transformaciones integran crecientemente las observaciones que la sociedad establece sobre sí misma a través de las ciencias sociales. C omo señala Giddens: «la sociología y las ciencias sociales en el sentido más amplio son elementos inherentes de la reflexividad institucional de la modernidad» (G iddens, 1995b: 10). La sociología es apropiada por los sujetos.

La reflexión sociológica de los últimos años coincide al recoger un aspecto en las sociedades avanzadas que, hasta no hace mucho, apenas había sido percibido: el desarrollo de la reflexividad institucional (Giddens, 1995c: 114). La sociología observa la inserción de las propias aportaciones de la investigación social en la conciencia y práctica de la sociedad estudiada. Inserción que, en nuestro momento mass-mediático, es subrayada por el éxito de público de al gunos autores pensadores de lo social 8 . Los sujetos se muestran crecientemente atentos a los análisis de las tendencias colectivas y adaptan sus conductas en función de las predicciones hechas sobre el futuro.

Ahora bien, si se asume la reflexividad a partir de los resultados de la investigación social, estamos obligados a aceptar la reflexividad en el propio proceso de producción de los resultados de la investigación, en la medida que los sujetos intervengan activamente en tal proceso. Los sujetos se comportan en la sociología empírica en función de la percepción de la demanda de ésta y de la demanda de la sociedad sobre ésta, es decir, según vean afectados sus intereses por los posibles resultados de la misma y las instancias que pudieran apli-

7. Véase el cuadro I, en el que se proponen los tres tipos de sociedad que concretan la modernidad (industrial, de consumo y de comunicación) y sus implicaciones en el papel del sociólogo y de la investigación social.

8. Véanse los ejemplos de M arcuse, Fromm, G albraith, $D$ aniel Bell, etc., por no citar la extensa divulgación de los clásicos - teniendo como ejemplo la obra freudiana - en la época de la divulgación y la sociedad del conocimiento, como la denomina, entre nosotros, Lamo de Espinosa (1990, 1994). El proceso inverso también se produce: autores, que aparecen como pensadores de lo social, que producen obras destinadas, casi en exclusiva, a satisfacer al gran público. 
car tales resultados. Al igual que adaptan sus comportamientos a las predicciones - pudiendo quedar éstas como profecías autocumplidas (M erton, 1972) o como predicciones autodestructivas (M erton, 1980) - los sujetos que participan en la investigación social adaptan su comportamiento durante la observación a la previsión de los resultados y las consecuencias de los resultados de la observación. Es decir, se trata de sujetos en la situación de investigación social desbordando continuamente su estatus de objeto, aun cuando sea sólo esta posición como objetos la que domina en el dispositivo de registro/análisis (encuesta, entrevista o grupo de discusión). Son sujetos que en su comportamiento incorporan su posición en relación con la instancia observadora, de manera que hasta la aceptación de su papel como objetos de observación - el «iy esto para qué sirve?» del final de muchos grupos de discusión- Ileva implícito su carácter de sujetos, pues aceptan pasar por objetos en cuanto suje tos. Un ejemplo como el siguiente, obtenido a partir de la reflexión que establece el propio grupo, al final del mismo, sobre la experiencia experimental, subraya la presencia de dimensiones pragmáticas sobre la macrosituación ${ }^{9}$ durante la dinámica: «H ombre pues si puedes Ilegar, con esto que estamos hablando, si puedes llegar un poquito alto, a decírselo por ahí a cualquiera, pues a mí me parece muy bien ${ }^{10}$. Circunstancia repetidamente recogida en la dinámica grupal y que presiona el nivel metodológico hacia la asunción, en la medida de las posibilidades de la investigación concreta, de esta subjetividad estratégica con respecto a la propia investigación, que incluye la percepción de los fines y quehaceres de las ciencias sociales. Por lo tanto, circunstancia que presiona hacia cierta dialectización de la investigación social, hacia un recorrido en el eje de las perspectivas metodológicas desde la perspectiva distributiva y estructural hacia la dialéctica: pues, hasta en su objetivización, los observados se atraviesan como sujetos, dejando de ser meros soportes de discursos.

En la observación de la sociedad, la jerarquía del analista sobre el receptor de sus resultados parece rozar su fin, al igual que otras relaciones jerárquicas'11.

9. Se establece la diferencia entre macrosituación o campo social objeto de observación y la microsituación o contexto inmediato de producción de datos o producción discursiva.

10. Fragmento discursivo de una reunión de grupo entre trabajadores autónomos en Alcalá de H enares, en octubre de 1994. Para más detalles sobre el estudio en el que se engl oba esta reunión, véase C allejo 1996. En este aspecto, los participantes en los grupos suelen creer más en la eficacia de la investigación que el propio investigador: «H ombre, y yo creo que de algo servirá, no a corto plazo... » (reunión de grupo de jóvenes entre 14 y 16 años, M adrid, febrero de 1996). Si está en su creencia ¿por qué no aceptar que las hipótesis de eficacia de los resultados de la investigación guían la actuación de la misma?

11. C aracterizando la actual sociedad, dice Lyon: «L a jerarquía se derrumba bajo la presión de las nuevas técnicas de gestión - en el Silicon Valley, las camisetas y los vaqueros sustituyen a los trajes formales- de los movimientos de mujeres, de una nueva conciencia étnica y sexual. Los intel ectual es son degradados de tareas legislativas a interpretativas, de la misma manera que el seminario ha sustituido a la clase tradicional en la universidad. En vez de imponerse los proyectos de los expertos, en la arquitectura y la planificación urbana se consulta con los clientes, y el clero fomenta una mayor participación de los "legos" en la iglesia» (Lyon, 1996: 110). 
No cabe reservar al observador unas condiciones esencial mente distintas a las del observado: Ios procesos de subjetivización y objetivización, que son los que ponen en marcha un proceso de reflexividad continuo, se encuentran en ambos lados de la relación de observación. Reconocimiento de unos sujetos con conciencia de tales ${ }^{12}$, de ser partícipes de una sociedad y sus destinos, aun cuando sea desde la perspectiva de la fatalidad, como ocurre en una parte importante de los sectores populares de la sociedad.

Siguiendo a autores como Giddens (1995a, 1995b), Beck (1992) o Lamo de Espinosa (1994), la modernidad ha entrado en una fase que cabe calificarla de reflexiva, en la que los sujetos tienen en el conocimiento científico una de sus referencias básicas con las que juzgar las situaciones ${ }^{13}$. Beck formula esta idea en su análisis de la conciencia que ha tomado la sociedad con respecto al medio ambiente, campo en el que los pronunciamientos científicos ocupan un lugar paradigmático: señalan las causas de peligro y los niveles a partir de los que cabe acentuar las precauciones. Pero en el caso de la ciencia social ha de darse un paso más, pues la sociedad no sólo aparece como receptora de resultados sino como participante en la creación de tales resultados.

Ahora bien, como el propio Beck (1992: 58 y s.) pone de manifiesto, la aproximación al conocimiento científico conlleva tanto: a) la adjudicación a éste de una mayor capacidad política; como b) la desmitificación de la ciencia a partir de la repetida vivencia de la escasa predecibilidad de las consecuencias de sus acciones y de al gunos efectos «mostruosos». Al centrar más su posición en el campo político, la ciencia se abre a las posibilidades de ser deslegitimada desde fuera de la ciencia. Proceso que todavía es incipiente, pero del que no faltan ejemplos: los expertos garantizan la seguridad de los residuos atómicos bajo suelo rocoso (granito), sin embargo, la reacción en contra de emplazamientos de cementerios nucleares de la población de las local idades cercanas es inmediata. Siguiendo de cerca al propio Beck, la gente utiliza su conocimiento - sobre todo, centrado en la presencia de errores y de riesgoscontra la ciencia: «la ciencia empieza a extender el poder metodológico de su escepticismo sobre sus propios fundamentos y resultados prácticos» (Beck, 1992: 156), caracterizando la sociedad: «por su escepticismo generalizado respecto a las razones providenciales ligado al reconocimiento de que la ciencia y la tecnología tienen un doble filo y crean nuevos parámetros de riesgo, al tiempo que ofrecen posibilidades beneficiosas para la humanidad» (Giddens, 1995b: 43). Por lo tanto, los sujetos observados pueden utilizar su conocimiento como «sociólogos espontáneos» o su escepticismo contra la aplicación concreta de la sociología, desconfiando de tales aplicaciones. Con ello, se entra en la política de la ciencia, al go que ha estado siempre en el centro cuando se enfrenta la reflexividad en la investigación social empírica.

12. Beck sitúa como causa de la modernidad reflexiva el proceso de individualización de las sociedades avanzadas. No es cuestión de discutir aquí qué fue antes, si la conciencia de suje to acrecienta la individualización y la reflexividad 0 , al contrario, como propone el autor alemán. 
La manera que cabe considerar tradicional en la que se introduce la política en la ciencia social, es la consistente en la concepción de sectores sociales dominantes utilizando la investigación para reproducción de la situación de dominación, haciendo de los investigadores, como del conjunto de los intelectuales, esa fracción dominada de la clase dominante (Bourdieu, 1988: 294). No obstante, hay campos en los que las relaciones de dominación están compuestas por un difícil equilibrio entre los distintos agentes en conflicto. Campos donde se requiere la elaboración de un consenso entre tales agentes para la construcción y aceptación de políticas concretas. Es aquí donde se abren las posibilidades para nuevas concreciones de prácticas de investigación. Siguiendo el argumento, el potencial de tales prácticas estaría en su capacidad de asumir la reflexividad ontológica y epistemológica y proyectarla en los niveles metodológico y técnico, al qué hacer en la investigación concreta, para que la reflexividad tenga un lugar protagonista. Reflexividad metodológica que tiene la obligación de contar tanto con la reflexividad en la aplicación de los resultados de la observación, como la que se produce en la propia observación.

Por supuesto, no es la primera vez que se apela a una sociología empírica reflexiva. D e hecho, la teorización mertoniana sobre la mediación de la toma de conciencia de las predicciones sociológicas en la acción social colectiva, que cabe señalar como uno de los primeros trazos relevantes sobre la cuestión, se reconoce deudora de buena parte del pensamiento social precedente (M erton, 1980: 173). D e forma manifiesta, está en los textos de G ouldner (1970) o la antropología que empieza a ser postmoderna hacia los años sesenta (Clifford, 1995: 106; Ruby, 1992; Clifford y M arcus, 1991; Geertz y otros, 1991). Puede encontrarse en Gouldner, como parte de su propuesta sociología radical: «radical porque advertiría que no es posible avanzar en el conocimiento del mundo si el sociólogo no se conoce a sí mismo y a su situación en el mundo social» (Gouldner, 1970: 444) o «una "sociología reflexiva" se ocupa, primeramente y ante todo, de lo que los sociólogos quieren hacer en el mundo y de lo que en realidad hacen» (Gouldner, 1970: 443). Incluso puede advertirse esta tendencia en las posiciones del socioanalista Loureau (1970) y su concepción de la implicación del sociólogo. Una sociología reflexiva más atenta a la subjetividad del sociólogo que a la de los observados. La diferencia básica de tales propuestas con la sociología reflexiva que se propone en la actualidad consiste en el carácter ontológico que ésta otorga a la refle-

13. La sociedad ha sido siempre reflexiva. H oy se asume tal reflexividad. Los antropólogos, entre las ciencias sociales, no han dejado de mostrar la resistencia de los observados a la observación. Era muy evidente para ellos y su trabajo de campo. Lo que ocurre ahora, más allá de que los subordinados muestren resistencias desde su propia cultura, lo que ha estado siempre presente, es que tal resi sencia se hace desde instrumentos tomados de la propia ciencia. Al menos, desde la propia concepción de la ciencia. Antes era una lucha entre combatientes desigual mente desarmados. H oy, el propio éxito de la ciencia ha dispensado sus propias armas en una extensa capa de la sociedad. La reflexividad social parte de que todos somos expertos. 
xividad de la sociedad (Giddens, 1984, 1995a, 1995b; Beck, 1992; Beck, Giddens y Lash, 1994; G arcía Sel gas, 1994). Así, la asunción de la reflexividad ontológica está más cerca de M erton que de la reflexividad narcisista que se propone como guía de trabajo empírico.

La sociología de la sociedad industrial apenas asume la reflexividad (tabla 1). El sociólogo ve el mundo. Razón por la cual los textos de los clásicos de la sociología tienden a situar en lugares y textos distintos sus reflexiones metodológicas y sus análisis de la sociedad: la observación no condiciona lo observado. La sociología de la sociedad que se ha denominado de consumo reconoce la necesidad de transformar la mirada sociológica. Transformación que se sitúa en el horizonte de la transformación del mundo; pero, cabría decir, sin el mundo: el sociólogo transforma el mundo - posición de Gouldner14_ y se transforma como sociólogo - posición L oureau- . Propuestas más destinadas a llamar la atención sobre la necesidad de «vernos como a otros», con lo que tiene más de objetivización de la subjetividad sociológica desde el propio observador que de asunción de la subjetividad de los observados. L os críticos autores de la sociedad de masas, privaban a ésta de cualquier imaginación sociológica. Tales sujetos observados no poseen la cualidad reflexiva: «no poseen la cualidad esencial para percibir la interrelación del hombre y la sociedad» (Wright M ills, 1993: 22). Sin embargo, si algo ha cambiado en la reflexión sociológica es la creciente asunción de una reflexividad ontológica.

El reconocimiento del carácter especular - y especulativo- de la sociedad ha tenido su reflejo en la teoría social. D e hecho, buena parte de las propuestas teóricas de la aplicación de la autodenominada ci bernética de segundo orden al estudio de lo social se erigen sobre la base de la reflexividad/retroalimentación. No obstante, cuando se llega a la investigación social empírica, el constructivismo hace más hincapié en la producción subjetivista de los observadores ${ }^{15}$, que en la subjetividad de los observados, pues si se parte de que la observación produce lo observado, también lo será la hipotética subjetividad de los observados. La subjetividad de los observados queda nuevamente subsumida, esta vez en la subjetividad del observador. El ojo del observador termina por no recoger el ojo observado, parafraseando el título de una obra que sirve de homenaje a Von Foerster, padre del constructivismo (Watzlawick y Krieg, 1994). D e hecho, desde este programa metodológico, toda intersubjetividad puede reducirse al interior del observado. Se crea una broza de bucles en la que los observados acaban perdiendo el carácter de sujetos en el proceso de observación.

14. La reflexividad de Gouldner, como afirma Bourdieu (1992: 51), es más un plan programático que un método de trabajo.

15. Para la relación entre constructivismo, reflexividad e investigación, aun cuando se echa de menos una mayor concreción empírica en el campo de lo social, véase alguno de los trabajos compilados por Steier (1991). Para una aproximación a la relación entre constructivismo, reflexividad y metodologías cualitativas, véase N oya (1994). 
Tabla 1

\begin{tabular}{|c|c|c|c|}
\hline & $\begin{array}{l}\text { Sociedad } \\
\text { industrial }\end{array}$ & $\begin{array}{l}\text { Sociedad } \\
\text { de consumo }\end{array}$ & $\begin{array}{l}\text { Soc. de comunicación (reflexiva) } \\
\text { - M omento de śntesis }\end{array}$ \\
\hline $\begin{array}{l}\text { M odelo } \\
\text { capitalista }\end{array}$ & $\begin{array}{l}\text { Capitalismo de } \\
\text { producción } \\
\text { - Plusvalía } \\
\text { en el trabajo }\end{array}$ & $\begin{array}{l}\text { Capitalismo } \\
\text { de consumo } \\
\text { - Producir } \\
\text { para consumir }\end{array}$ & $\begin{array}{l}\text { Capitalismo especulativo } \\
\text { - Unos «juegan» } \\
\text { con otros globalmente } \\
\text { - Consumir para producir }\end{array}$ \\
\hline Lógica social & $\begin{array}{l}\text { Atomista } \\
\text { (individuos) }\end{array}$ & $\begin{array}{l}\text { Relacional } \\
\text { - Individuos con } \\
\text { individuos } \\
\text { - «nfluencia personal» }\end{array}$ & $\begin{array}{l}\text { Reflexiva } \\
\text { - Sujetos en sujetos }\end{array}$ \\
\hline $\begin{array}{l}\text { Relaciones de } \\
\text { dominación }\end{array}$ & $\begin{array}{l}\text { H eterocontrol } \\
\text { orgánico } \\
\text { - División del } \\
\text { trabajo }\end{array}$ & $\begin{array}{l}\text { H eterocontrol grupal } \\
\text { - Comunidad de } \\
\text { consumidores }\end{array}$ & $\begin{array}{l}\text { Autocontrol } \\
\text { - Redes nudos, } \\
\text { experiencia interna }\end{array}$ \\
\hline $\begin{array}{l}\text { Formas del } \\
\text { consenso }\end{array}$ & $\begin{array}{l}\text { Consenso mecánico } \\
\text { - M asas } \\
\text { - Efectos }\end{array}$ & $\begin{array}{l}\text { Forzar el consenso } \\
\text { - La necesidad del } \\
\text { consenso } \\
\text { - Guerra fría } \\
\text { - Pactos } \mathrm{E} \text {. bienestar }\end{array}$ & $\begin{array}{l}\text { Consenso en el disenso } \\
\text { - Consenso desde la conciencia } \\
\text { de disenso } \\
\text { - D erecho a discrepar, a diferir }\end{array}$ \\
\hline $\begin{array}{l}\text { Relación con } \\
\text { el trabajo }\end{array}$ & O ficio/vocación & $\begin{array}{l}\text { Evasión como } \\
\text { imaginario } \\
\text { Centralidad oficio }\end{array}$ & $\begin{array}{l}\text { Reciclaje } \\
\text { - De trabajo } \\
\text { - De cosas }\end{array}$ \\
\hline $\begin{array}{l}\text { Conexión con } \\
\text { la naturaleza }\end{array}$ & $\begin{array}{l}\text { Rapiña } \\
\text { - Colonialismo } \\
\text { - Cosas }\end{array}$ & $\begin{array}{l}\text { Absorción } \\
\text { - O bjetos de } \\
\text { destrucción }\end{array}$ & $\begin{array}{l}\text { Reciclaje } \\
\text {-Ecológico } \\
\text { - Feed-bach } \\
\text { - Autocontrol «para otros» }\end{array}$ \\
\hline $\begin{array}{l}\text { Fuente de } \\
\text { identidad }\end{array}$ & El origen y la clase & $\begin{array}{l}\text { Posición estructural } \\
\text { - Estilo de vida } \\
\text { (corrientes } \\
\text { socioculturales) }\end{array}$ & $\begin{array}{l}\text { D iferenciación minimalista } \\
\text { - Estilos de vida en } \\
\text { minimalización } \\
\text { - La minimalización de la gama } \\
\text { - Espejo de posiciones }\end{array}$ \\
\hline \multirow[t]{2}{*}{$\begin{array}{l}\text { Perspectiva } \\
\text { sociológica }\end{array}$} & $\begin{array}{l}\text { D istributiva } \\
\text { - Encuesta }\end{array}$ & $\begin{array}{l}\text { Estructural } \\
\text { - G rupo } \\
\text { - Análisis } \\
\text { correspondencias }\end{array}$ & $\begin{array}{l}\text { D ialéctica reflexiva } \\
\text { - Teoría de redes } \\
\text { - Socioanálisis global } \\
\text { - Multilevel } \\
\text { - Evaluaciones continuas } \\
\text { (de la investigación) }\end{array}$ \\
\hline & Sujeto/objeto & O bjeto/sujeto & $\begin{array}{l}\text { O bjeto en el sujeto y } \\
\text { sujeto en el objeto }\end{array}$ \\
\hline $\begin{array}{l}\text { Lógica de la } \\
\text { observación social }\end{array}$ & $\begin{array}{l}\text { Explotación } \\
\text { (producción de datos) }\end{array}$ & $\begin{array}{l}\text { Comparación } \\
\text { (reproducción de datos) }\end{array}$ & $\begin{array}{l}\text { Don } \\
\text { (reflexión de datos) }\end{array}$ \\
\hline $\begin{array}{l}\text { H acer } \\
\text { del sociólogo }\end{array}$ & $\begin{array}{l}\text { M edir } \\
\text { - Estímulos } \\
\text { - Poblaciones }\end{array}$ & M anipular & Enfrentar posiciones \\
\hline $\begin{array}{l}\text { Intención } \\
\text { sociológica }\end{array}$ & $\begin{array}{l}\text { D escribir situaciones } \\
\text { - Sociología } \\
\text { administrativa }\end{array}$ & $\begin{array}{l}\text { Explicar procesos de } \\
\text { influencia } \\
\text { - Sociología crítica }\end{array}$ & $\begin{array}{l}\text { Promover procesos } \\
\text { - Sociología reflexiva } \\
\text { - ¿Cómo desconectar a } \\
\text { los autocontrolados? }\end{array}$ \\
\hline
\end{tabular}




\section{Campos de actuación preferente de la articulación de perspectivas}

El consenso es un proceso que se construye discursivamente. La reflexividad y el consenso se producen a través de discusiones (Giddens, 1995a: 56). Reflexividad y consenso se alimentan recíprocamente a través de discursos. La propuesta de programas de acción concretos puede tener una doble relación con la investigación de los discursos. En primer lugar, investiga los discursos para obtener de ellos la propuesta del programa, como una especie de mínimo común denominador para establecer las bases de la acción. A hora bien, la relación puede establecerse a partir de una propuesta concreta de acción - un programa de acción - con el objetivo de observar cuál podía ser su recepción entre los diversos agentes y, además, establecer las modificaciones adecuadas hacia el logro del más extenso y profundo consenso con respecto a tales programas de acción. Profundidad del consenso que implica la vinculación con el mismo y con los discursos que lo sostienen, por parte de los sujetos. Ambos tipos de relación están muy conectados entre sí, de modo que, en la aplicación práctica de la investigación, cabe la posibilidad de abordarlos conjuntamente: la circulación de discursos sobre posibles programas de acción y recepción/vinculación de algunas propuestas de acción concreta.

El material que se ha de recoger se encuentra en campos caracterizados por rasgos como:

a) Presencia de agentes en conflicto.

b) Existencia de circulación de discursos, lo que lleva a dispositivos de investigación social pertinentes a tal circulación.

c) Posibilidades de negociación de políticas concretas de acción, lo que requiere también observar la vinculación a los posibles acuerdos.

d) Conciencia de los agentes sociales como tales agentes, con un relativo grado de consenso interno en los grupos sociales en el campo de actuación. $\mathrm{H}$ a de existir cierta conciencia de grupo y, por lo tanto, reconocerse cierta presión del grupo, frente a posibles tendencias individual istas.

Rasgos que condicionan un tipo de material que, en primer lugar, inclina la investigación hacia dispositivos flexibles, en los que el discurso pueda apuntar hacia el diálogo, sólo posible en microsituaciones de relación horizontal y no en la jerárquica situación de observados/observadores. La microsituación (contexto grupal o inmediato16) ha de apuntar a cierto isomorfismo con la

16. Tiene razón N oya (1994) cuando critica el uso del término contexto por los cualitativistas. Pero ¿qué es el contexto en lo social? En sentido estricto, más allá de referencias al contexto como descripción de la macrosituación o la microsituación, no hay contexto en lo social. Por ello, parece conveniente, especialmente para el caso del grupo de discusión, hablar más de intertextualidad que de contextos, lo que supone la necesidad de articular reflexividad con el paradigma dialógico. Para una extensión de la concepción dialógica a la metodología de las ciencias sociales, véase la compilación de G uba (1990). Exposiciones sobre la concepción dialógica del grupo de discusión se encuentran en Vallejos (1995), Callejo y Vallejos (1996) y Alonso (1996). 
macrosituación, como medida de apoyo a la simulación: horizontalidad de los sujetos que ocupan una misma posición en el campo en conflicto, cuando hablan y se relacionan entre ellos.

En segundo lugar, inclina la investigación hacia prácticas en las que el habla de los sujetos tenga un papel principal, por lo tanto, hacia prácticas de investigación conversacional es. En tercer lugar, hacia marcos de observación donde los sujetos investigados puedan sentirse directos sujetos de acción, aun cuando sea imaginariamente. El cumplimiento de estas tres condiciones es lo que abre la propuesta de realización de grupos de discusión 17 a la manera de lo que los sociólogos y psicólogos institucionales de final es de los años sesenta llamaron soci oanálisis (perspectiva dial éctica), lo que implica la hipótesis de asumir el campo particular de lo social como una especie de macrosituación organizacional, donde la gestión en el consenso parece posible18.

La aplicación del socioanálisis, técnica paradigmática de la perspectiva dialéctica, tiene en las organizaciones su campo privilegiado. Se trata de organizaciones que se hacen conscientes de cierta esclerosis, en medio de un conflicto, y buscan dinamizarse, en lo que tiene de retomar nuevas energías, como de adaptación a nuevos retos. C omo subraya Loureau $(1970,1977)$, el socioanalista interviene en una situación de conflicto. Toma de consciencia que tiende a proceder de una fal ta de consenso dentro de la organización y un déficit de legitimación de sus objetivos, lo que cabe entender como un déficit de legitimación de la propia organización desde su interior. Por lo tanto, el encuentro con la expresión del disenso y la búsqueda de las posibilidades de consenso están en su centro.

El modelo de sociedad que está detrás del socioanálisis es el de una sociedad institucionalizada, fuertemente burocratizada, que ha reducido los sujetos a objetos, cayendo en la contradicción de que mientras su eficacia depende de tal reducción de los sujetos a mecanismos de la organización, es incapaz de reproducirse de manera ampliada, pues la ampliación, a partir de la reincorporación de energía, sólo es posible a partir de los sujetos. D e lo contrario, únicamente se produce ampliación de la esclerosis burocrática. Si la reinversión de la plusvalía amplía la base material del capital (capital constante, en

17 Aun cuando en este escrito, la referencia de reuniones de grupo se establece sobre su fórmula más habitual en España, con al rededor de ocho participantes, cobra especial sentido, para la investigación de aceptación de programas de acción, su articulación con los grupos triangulares (tres participantes), siguiendo la propuesta de Fernando C onde (véase el capítulo «Los grupos triangulares como "espacios transicionales" para la producción discursiva» en Conde, 1996).

18. Es aquí donde se apoyan metáforas como la que asimila una nación a una organización empresarial privada, sobre todo si se observa cómo los ciudadanos asumen el papel de empleados contribuyentes que esperan una retribución compensación directa, los partidos políticos o similares toman las características de comités de representación y la discusión sobre los presupuestos, a veces de anual es convenios colectivos donde se concentra la principal conflictividad social, cuestión que fue adelantada por D aniel Bell (1976) en su perfil de la sociedad posindustrial norteamericana. 
términos marxistas), la reinversión de la subjetividad amplía la base organizacional19. Además, tal reducción a objetos de los sujetos no se logra totalmente sino que topa con la resistencia de los sujetos que, con conciencia de alienación, proyectan su subjetividad fuera de la institución, ya sea en ámbitos ajenos a ésta 0 , incluso, contra ésta. El objetivo institucional último, para lo que reclama el socioanálisis, es para rerracionalizar la institución superando tal contradicción, buscando la resubjetivización de la integración de los sujetos. Esta es la clave de la reforma que mantiene como objetivo. D e aquí que el socioanálisis pueda servir de modelo para el sondeo de la implicación con programas de actuación, aun cuando se sal gan del ámbito institucional organizacional: hay una explícita búsqueda de reinversión de subjetividad para plantear políticas que cuenten, ya no sólo con legitimación, sino también con la participación de los sujetos.

En su forma, el socioanálisis toma las características de un proceso asambleario con el horizonte de un cambio radical. Es más, su operatividad ha de tener su origen en un objetivo de liberación por parte de los sujetos que participan en el mismo (Lapassade, 1971: 58). Sin embargo, su horizonte es la reforma de la organización, lo que ya conlleva, aun cuando de manera difusa, un posible programa de actuación. Si el potencial del grupo de discusión está en su horizonte reformista, para el que invoca a los sujetos; el potencial del socioanálisis está en su horizonte revolucionario.

El posible grupo que se establece al rededor de la experiencia socioanalítica es un grupo sujeto desde el principio. Los sujetos participantes en la experiencia socioanalítica son los portadores y operadores del discurso que producen. Esto le diferencia del grupo de discusión, donde el discurso de los participantes en la reunión es enajenado para ser gestionado por otros. La apertura que aquí se establece con respecto al grupo de discusión es que, dado que se necesita la participación de los sujetos para que la gestión final tenga alguna posibilidad de éxito, ha de situarse el grupo y sus sujetos en situación de participación en la gestión 0 , al menos, de participación en los out-put de la gestión.

¿Para qué condiciones concretas de la investigación (macrosituación) es preferente la articulación de las dos perspectivas (estructural, dialéctica) y, en al guna medida, la inclinación de la reunión de grupo a adquirir, en su dinámica, rasgos socioanalíticos? Sobre todo, parece particularmente pertinente en aquellas investigaciones que buscan sondear las vinculaciones con futuras pro-

19. De esta manera, dimensiones como la creatividad y la capacidad imaginaria de los sujetos, al reinvertirse en la organización, tienen menos posibilidades de salirse del sistema, de proponer alternativas al sistema, con el previsible resultado de que cuanta más energía se reinvierta en el sistema organizacional o campo social, menos queda para plantear soluciones globales al propio sistema. H ipótesis apoyada en el modelo marxista, en la medida que mantiene que la necesidad de reinvertir en capital constante (maquinaria y tecnología, por ejemplo) la plusvalía generada por el trabajo conduce a situaciones futuras en las que será más difícil obtener plusvalía, ya que crecientemente hará falta emplear menos trabajo (capital variable) en relación con el capital constante. 
puestas o programas de actuación, que van a exigir de la participación de los sujetos y que parten de situaciones conflictivas, donde los sectores social es observados ya han tomado posiciones como agentes. Es decir, macrosituaciones en las que haya que contar con la activa participación de los diversos agentes y, sobre todo, de los sujetos 20 .

En primer lugar, hay que señalar que se trata de una investigación de campo, en relación directa con los sujetos, que se va a mover entre la apariencia formal del grupo de discusión y la línea imaginaria de un socioanálisis sin límites organizacionales precisos, sin organización (nivel medio-microsocial) y sin revolución (nivel macrosocial).

En segundo lugar, que este tipo de investigaciones tienen su origen en un acuerdo explícito 0, al menos, en un reconocimiento mutuo entre los principales agentes institucionales en un campo de prácticas determinado. Por ejemplo, organizaciones empresariales, sindicatos y Administración, en el caso de un determinado campo productivo; asociaciones de distintas categorías profesional es y usuarios en, por ejemplo, campos como la medicina, la justicia, el comercio, o distintos agentes (Administración, sindicatos, empresarios, trabajadores, movimientos sociales) en el caso de políticas medioambientales, etc. Principio de acuerdo que sitúa al sociólogo investigador en una especie de papel arbitral, de agente externo dispuesto para apaciguar conflictos, si la situación es caliente, o como inicio del consenso en la búsqueda común de acuerdos para la aplicación de medidas concretas. El consenso entre los agentes está en la conciencia de que las medidas, en principio, son necesarias. Las diferencias están en su aplicación.

Los agentes presentes en el campo se reconocen entre sí. D e aquí que cada uno de los grupos deba tender hacia una mayor homogeneidad social de la que se da en otro tipo de estudios, que puedan reconocer las contradicciones que surgen en el grupo como contradicciones propias y no como el reconocimiento de posiciones sociales distintas. Como tal conciencia de agentes sociales, se facilita la percepción del objetivo del estudio: la búsqueda de un consenso para establecer acciones comunes. Como agentes plantearán sus intereses en el consenso, así como los obstáculos.

En la medida que hay un horizonte de búsqueda de consenso, los sujetos, como agentes sociales, toman como referencia las posiciones de otros agentes presentes en el campo. Reconstruyen, desde su perspectiva a los otros agentes y, así, se reconstruyen a sí mismos como tales. Reconstrucción que es importante para observar la distancia respectiva proyectada y, por lo tanto, la percepción de puntos de acuerdo.

Con respecto a la investigación más convencional con grupos de discusión, supone algunos cambios. La mayor diferencia se encuentra en el papel del

20. Se establece la distinción entre agentes, posiciones diferenciadas en un campo social y sujetos, reflejando en éstos su potencial individualizador. Los sujetos pueden actuar como agentes, desde la conciencia de una posición colectiva en un campo social, o como individuos, estableciendo sus estrategi as concretas supuestamente al margen de posiciones colectivas. 
moderador, ya que, producto del acuerdo que da pie a la investigación, representa las posibilidades de intereses comunes y, en definitiva, el conjunto de intereses. D e aquí que evite al máximo el acudir marcado al grupo o ser marcado por el mismo, para así no encontrar enfrentamientos o complicidades. Lo ideal es que los enfrentamientos y complicidades, entre los participantes y con el campo de estudio, sean interiores al grupo producto del diálogo intragrupo y extragrupo y no producto de la observación; pero ya sabemos que la observación está marcada por la situación de observación, aun cuando intente desmarcarse el observador. N o pudiendo escapar del marcaje por la huida, creándose una especie de lugar vacío, recomendado en la moderación de grupo (I báñez, 1979), la alternativa está en constituirse como propio escenario del consenso.

D e hecho, a quien dirige la intervención socioanalítica no puede dársele el calificativo de moderador, como ocurre con respecto al grupo de discusión. Le ajusta mejor la categoría de agitador, procurando poner al grupo contra sí mismo. Si la no directividad grupal apenas obtiene frutos en campos con discursos muy cristalizados o posiciones de los agentes al ejadas del consenso, es decir, situaciones con escasa inversión de subjetividad, la agi tación moderada cobra todo su sentido para crear escenarios de propuesta de nuevos discursos y nuevos escenarios: poner al grupo contra sí mismo es la mejor manera para entrar en contacto con las implicaciones subjetivas del grupo, tanto en la microsituación (grupal) como con respecto a la macrosituación. Entre la provocación y la no directividad hay un amplio margen en el que se juega la aproximación de la perspectiva estructural a la perspectiva dialéctica. Una aproximación que replantea parcialmente la manera de entender el grupo de discusión cuando se intenta trabajar con las inversiones de subjetividad de los observados y no sólo como soportes de discursos. Pero, como ocurre con toda introducción en la dinámica grupal, tal cambio conlleva un mayor acento en los problemas de método (O Imsted, 1992: 77). Problemas que aparecen en un proceso cuya finalidad es no acabar, pues, desde el punto de vista del sociólogo, los grupos de discusión no interesan como grupo sino como agrupación ${ }^{21}$. D e aquí la necesidad de que el moderador evite, con sus intervenciones, la constitución de grupo como tal: los sujetos y la reflexión sobre su vinculación grupal aparecen más fácilmente cuando no hay un grupo constituido como un único sujeto.

\section{El papel del moderador}

Con respecto a su dinámica, en el socioanálisis destacan más los agentes que sus fases, pues no puede hablarse de fases. Se establece sobre la competencia entre los líderes que se sitúan bajo el líder socioanalista. U na competencia que, en

21. De hecho, la experiencia que conforma la reunión de grupo no entra en ninguna de las clasificaciones al uso de los grupos: ni primario, ni secundario; ni grupo afectivo, ni grupo tarea, etc. 
el enfrentamiento, puede abrir la voz a los callados, a que los silenciosos tomen la palabra en favor de unos u otros. $D$ an pie a la queja de los silenciosos por subordinación 22 . D esde esta perspectiva, la reflexividad social está principalmente en los subordinados, como destaca Rosaldo: « os dominados usualmente comprenden al dominante mejor que al revés» (Rosaldo, 1993: 184). Presiona a los silenciosos por simulación: «toda estructura incluye un ámbito de simulación, y el socianálisis constituye un di spositivo de simulación que desvela lo disimulado» (I báñez, 1990: 75). Poner a los simuladores contra su simulación se convierte en una finalidad del agente socioanalítico.

Poner el grupo contra sí mismo, para observar su capacidad de llegar a un consenso más allá de la propia situación experimental del grupo de discusión, pues lo que se plantean son posibilidades de consenso entre agentes en un campo y la proyección operativa de tal consenso, exige una actuación por parte del moderador de la reunión que se ha caracterizado por la agitación moderada. Actuación que se separa de los cánones propuestos en la obra de I báñez sobre el papel del moderador, pues, en lugar de tender a la desaparición imaginaria, éste cobra especial protagonismo.

Poner al grupo contra sí mismo puede ofrecer a la observación el margen de flexibilidad de un sector social ante distintas situaciones o contrapropuestas; pero, entonces, se corre el peligro de que ya no se vea al investigador moderador como a un árbitro en un conflicto sino como el mensajero de otros agentes. Cuestión que no puede dejarse a un lado y que implica dos movimientos tácticos importantes:

\section{En la dinámica de la reunión:}

a) Establecer la agitación tras la aproximación, tras haber dejado al grupo la exposición de su posición e incluso haber dado algún signo de, al menos, comprensión de tal posición, como esfuerzo de colaboración en la construcción de su identidad. Sólo tras haber dejado al grupo una reconstrucción relativamente sólida de su identidad, cabe enfrentarle a la misma. Un enfrentamiento que puede establecerse desde la simulada alianza del moderador con las posiciones menos consistentes o más periféricas del grupo y, sobre todo, reclamando la participación de los que menos han intervenido. Así, el moderador investigador no aparece como uno de «fuera» sino como una «prolongación» de lo dicho dentro, con cierto afán individualizador bajo la pregunta sobre si la agrupación producida en la microsituación es consistente frente a los procesos de individualización, y la hipótesis de que la fuerza que retoman dinámicas individualizadoras es un indicativo de la capacidad de vinculación de las individualizaciones - siempre huidizas desde la perspectiva de los agentes- por parte de los agentes presentes en el campo social

22. N o es casualidad que en un texto sobre el sufrimiento, Bourdieu hable del socioanálisis (Bourdieu, 1991). 
de estudio. Es el momento en que el moderador colabora con los suje tos en la simulación de ruptura con su disimulación, con esas estrategias que desplegaron para enfrentarse a una situación de la que desconfian.

b) Utilizar principalmente propuestas de escenarios posibles, en los que poder encuadrar lo dicho por el grupo, bajo el argumento de que tal decir desea ser concretado para observar su proyección práctica. En una investigación destinada a ofertar cursos gratuitos de formación en seguridad e higiene en el trabajo, tal agitación moderada se establecía con intervenciones como: «habéis dicho que la salud es muy importante y que no se saben los peligros que hay para ella mientras se trabaja ¿qué es lo que estáis dispuestos a hacer para cambiar esto?, ¿qué se os ocurre?». D e esta manera se inicia el camino hacia lo que estaría dispuesto a ofrecer el resto de trabajadores o empresarios presente en la reunión.

c) Propuestas de escenarios posibles, que han de aparecer como «ocurrencia» de la propia dinámica. Sólo al final, con la propuesta de alternativas específicas, podría revelarse como propuestas prediseñadas. Frente a la situación del grupo (microsituación) a recrearse en su memoria (macrosituación), pues toda identidad se busca más fácilmente en el pasado, el moderador se convierte en una presión para echar el grupo hacia el futuro.

A pesar de las Ilamadas de I báñez (1979) sobre la inclinación de los grupos a constituirse en grupos sujeto, la experiencia dice que, especialmente entre las clases populares y las clases medias administrativas, la inercia a la subordinación adquiere una fuerza relevante. D e hecho, los participantes, cuya subjetividad está también presente en esta incorporación de su situación en la estructura social, vienen predispuestos a constituirse en un grupo objeto, pues, para ellos, es lo más asequible: «Sería un poquito más fácil, digo yo más asequible a [... ] no empezar con la conversación y dejarnos un poquito a nuestro aires 23 . Poco acostumbrados a declararse en la posición de sujetos, el moderador ha de provocarla, ya sea con sus tensos silencios o con sus intervenciones. Lo sujetos no pasan fácilmente a tomar esta posición en la experiencia grupal, pues, de hecho, se inclinan a la microsituación de pregunta-respuesta, orden-obediencia, siendo la proyección de su macrosituación estructural. La tendencia a constituirse en grupo objeto de al gunos sectores sociales se refuerza, además, por la satisfacción de los participantes en la cohesión y los acuerdos del grupo (Shaw, 1980: 266), lo que fácilmente lleva a grupos objeto con sujetos autoobjetivados, sobre todo si la macrosituación es muy conflictiva, con la presencia de importantes peligros externos para el sector social consultado, pues, entonces, la individualización queda casi bloqueada (Janis, 1974; Schachter, 1974). La labor del moderador agitador de obligar al grupo «contra sí mismo», incluyendo sus inercias prácticas, cobra nuevamente sentido. 
2. En el análisis del discurso producido, pues su sentido se ve trufado por las intervenciones del moderador. A partir del inicio de esta agitación moderada, el análisis no puede reducirse a las expresiones orales registradas en textos y sus cortes, pues la propia fuerza de la dinámica, los tonos de voz 0 el ritmo se establecen en indicadores tras poner al grupo contra sí mismo y las posibilidades de cambio, cambiando parcialmente ellos mismos, son la base para observar:

a) hasta qué punto el moderador observador ha pasado de ser observado como un técnico del consenso a serlo como portavoz de otros agentes o como mero agitador para la acción, como uno de ellos, en una asunción socioanalista del moderador;

b) las vinculaciones con las propuestas de escenarios posibles que se realizan para políticas concretas.

Como ocurre con la mayor parte de los factores y de las tácticas presentes en todas las prácticas de investigación, el margen que se abre tras un significante, como agitación moderada, es amplia. Pero no caben más recetas que las destinadas a señalar sus límites, entre un exceso de agitación, que llevaría la dinámica grupal a estar subordinada al protagonismo del moderador investigador, que encierra al grupo en la microsituación, y un exceso de moderación, que impediría revelar la vinculación del sector social representado en el grupo con políticas concretas o posibles escenarios, con lo que el grupo quedaría encerrado en la macrosituación.

\section{El reflexivo cierre dialéctico: la percepción de la sociología por el grupo}

El diálogo de los observados, presentes en el grupo, se establece también con el observador. Un diálogo que no cabe considerar entre el experto y el inexperto. ¿Q uién es el inexperto? ¿El sociólogo que no sabe de la experiencia de los sujetos observados o éstos que no saben sociología? Es un diálogo entre expertos en el que no cabe el «no saber». El sociólogo sabe un al go de la experiencia de los sujetos observados, con lo que especula. También, con lo que los sujetos observados especulan. Los sujetos observados saben del sociólogo como sociólogo y ellos mismos son sociólogos: «cada actor social competente, hay que añadir, es ipso facto un teórico social en el nivel de la conciencia discursiva y un especialista metodólogo en ambos niveles de la conciencia, el discursivo y el práctico» (Giddens, 1984: 18).

En el inicio de este escrito, se apelaba a la inserción de la percepción de la sociología por los sujetos en el mismo proceso de investigación, bajo la conciencia de que se verán implicados en los resultados. A hora bien, la dinámica se establece como agitación de la subjetividad de los participantes, como intento de ruptura con su adaptación a la concepción que tienen del quehacer sociológico. U na subjetividad presente, que se esconde como táctica (G offman, 1972, 1987) incorporada de presencia en microsituaciones consis- 
tentes ${ }^{24}$, que hace fal ta desvelar. Pero nuevamente el análisis tiene el encargo de rescatar tal percepción. El análisis de la subjetividad, por parte del observador, se convierte en la única vía para objetivar al sujeto objetivante (Bourdieu, 1992: 175), salvo que se disponga de una especie de observador del observador, distinto del observado, algo que ha de reconocerse como raro en la investigación social concreta habitual. Ello obliga a la observación de la relación, asumiendo así otro de los significados básicos de la reflexividad, como: «mirarse a sí mismo (self) en el yo-y-otro (self-and-other)» (Schechner, 1991: 40).

La inclusión, en el análisis del discurso producido por el grupo, de la media ción que realiza el moderador investigador, permite diferenciar dos tipos de mediación o asunción de la subjetividad del sociólogo en la subjetividad de los observados, confesándose el punto de partida, poco constructivista, de que tal análisis limpieza de la mediación del sociólogo moderador permitirá observar el objeto de estudio, la relación entre sujetos y agentes, con menos contaminación por parte de la microsituación de observación. El que el observador esté incluido en lo observado no reduce la observación al observador.

a) La mediación explícita. R eflejada, de manera manifiesta, en la transcripción de la reunión como intervenciones del moderador. $\mathrm{H}$ ay que reconocer otro tipo de mediaciones diluidas en gestos y movimientos que recoge el creciente uso de la cámara de vídeo en la reunión. Este trabajo del análisis conlleva romper con la tendencia a poner entre paréntesis la intervención del moderador en el propio análisis, sobre todo cuando coincide analista y moderador en una misma persona25.

b) La mediación implícita que se proyecta sobre las percepciones del sociólogo y la sociología, y el papel del mismo en el campo de prácticas abordado, poniendo de relieve la pregunta: ia quién sirve? Los sujetos hablan de una manera determinada en función de «a quién hablan» y, por lo tanto, cómo perciben al receptor.

Ambos aspectos tienen dos niveles: metodológico y sustancial, produciéndose un cuadro con las siguientes características.

- Dimensión sustancial de la percepción del sociólogo: a la sociología le interesa saber cuál es la percepción que tiene la sociedad de ella, sus prácticas y sus instrumentos, puesto que implica abrirse al reconocimiento de su reconocimiento y, por lo tanto, de sus posibilidades de actuación. En este nivel, se está en la sociología de la sociología, recomendada por Bourdieu (1992: 48), aun cuando ha sido pasada por la alteridad.

- Dimensión sustancial de la percepción del sociólogo en el campo de prácticas: en relación con el punto anterior, significa evaluar las posibilidades de actua-

24. Siguiendo a G rathoff (1990), cabe diferenciar entre situaciones socialmente consistentes y situaciones con un al to grado de inconsistencia social.

25. Tendencia del analista a ocultarse como productor de la observación. 
ción de la sociología en un campo determinado, pues no todos los cambios se abren con la misma facilidad a la intervención arbitral. Ello no deja de ser un síntoma sobre las posibilidades del consenso en tal campo.

- Dimensión metódica de la percepción del sociólogo: hasta qué punto lo dicho por los participantes en la reunión sobre el objeto de investigación está mediado con la percepción que tiene de la sociología. Así, una percepción mistificante y distante de la sociología puede inclinar el discurso a evasiones o retóricas hiperformales. U na percepción que se mueva en el otro polo, la sociología como práctica colaboradora, puede inclinar el discurso sobre la base de hacer del sociólogo «uno de los nuestros». La mayor potencialidad de la segunda para la observación de vínculos es manifiesta, precisamente porque revela la posición de sujetos de los participantes.

- Dimensión metodológica de la percepción del sociólogo en el campo de prácticas observado: consiste en evaluar hasta qué punto el diálogo grupal está mediado por el lugar que asignan al sociólogo entre los intereses en conflicto en el campo. Un grupo subordinado que percibe al sociólogo que interviene como instrumento de las posiciones dominantes, tenderá a comportarse y a hablar ante él como lo haría hacia quienes ocupan el poder.

La inclusión de la percepción que tiene el grupo del sociólogo no cierra la espiral de la reflexividad, pues ésta no tiene cierre. La propuesta de guía de análisis que se propone no deja de ser una incitación a la observación (al observador) de objetivar la subjetividad de los observados subjetivando al observando. Ahora bien, se adentra hacia su centro, con la conciencia de que estos pasos metodológicos refuerzan los análisis. Se trata de reconocer que en el propio proceso de conformación del grupo hay reflexividad, pues: «hay reflexividad cuando orienta mi acción lo que (creo que) el otro espera de mí» (Lamo de Espinosa, 1993), y que tal reflexividad metodológica se concrete en un útil relevante para la aplicación del grupo de discusión en ciertos ámbitos de la realidad social, pues acerca la observación a la vinculación de los sujetos con los discursos, al go especialmente importante en campos donde se generan con facilidad microsituaciones que empujan a estrategias conversacionales tipo «políticamente correcto».

\section{Conclusiones procedimentales}

La investigación sociológica inclina su operatividad desde la original confianza en el encuentro de la realidad al logro, actualmente necesario, de consensos sostenidos, incluyendo la percepción de tal realidad. En una sociedad en la que nadie tiene el monopolio de la razón, la investigación social ya no se dirige en busca de una «verdad sustancial» sino a la construcción de puntos de negociación entre los diversos puntos de vista: «en las definiciones de riesgos, el monopolio de la racionalidad de las ciencias está roto. $\mathrm{H}$ ay siempre demandas en competencia y conflicto, intereses y puntos de vista de los diversos agentes de la modernidad y grupos afectados, que están forzados conjuntamente 
en la definición de riesgos» (Beck, 1992: 29). Puntos de negociación que se convierten en claves en campos conflictivos y, también, en posibilidades de su futuro.

El grupo de discusión, emergido como práctica de investigación en la sociedad de consumo, tiene componentes que la hacen válida para una sociedad en busca de sus posibilidades y no sólo como depósito de memoria social. Es una validez desde la apertura, que tal vez haya que generalizar a las otras técnicas, aun cuando no todas las prácticas tienen la misma capacidad de apertura. A pertura que la capacita para ocupar un área donde se cruzan las perspectivas metodológicas estructural y dialéctica.

U na validez que implica un mayor esfuerzo en todas las dimensiones. Especialmente un esfuerzo de reconocimiento de la característica reflexivodialógica 26 del grupo de discusión. Si esta introducción en la reflevidad metodológica se ha centrado en la dinámica y el análisis del grupo de discusión, la caracterización dialógica va más allá de la propia dinámica. M ientras la reflexividad metodológica parte principalmente de la asunción de la reflexividad ontológica, el carácter dialógico del grupo de discusión integra tal reflexividad metodológica, para establecer esta técnica, como sugerencia procedimental, como un diálogo con:

- Los participantes en el grupo, ya sea a través de una mayor intervención del preceptor, lo que conlleva una subjetivización del interrogador para subjetivar al grupo.

- Los diversos grupos entre sí, con la devolución al grupo de la información producida por el propio grupo y por otros grupos previos.

- El análisis, pues se implica un diálogo con lo dicho por el grupo y el analista en la relación, donde ha de reconocerse.

D imensiones procedimentales que hacen de la asunción reflexivo-dialógica de la investigación social mediante grupos de discusión el elemento base de la articulación entre perspectiva estructural y perspectiva dialéctica. Elementos operativos que seguramente muchos investigadores sociales están Ilevando a cabo en sus prácticas concretas de investigación con grupos de discusión.

El descenso al nivel de lo técnico-metódico tiene un doble riesgo: el de una mayor implicación con los procesos concretos y sustantivos de la investigación y el de encontrarse con que la propuesta ya se está haciendo o que los investigadores ya están hablando en prosa sin saberlo. Pero si se desea proyectar uno de los temas más interesantes de la sociología actual, como es la reflexividad, en el trabajo empírico, no queda otra alternativa que aceptar tales riesgos. Por ello, tal vez haya merecido la pena resaltar al gunas implicaciones de esta asunción de la reflexividad en la práctica concreta de investigación, partiendo de la posibilidad de articular distintas perspectivas metodológicas. 
Elementos de procedimiento que han sido un intento de dar el paso desde la asunción teórica de la reflexividad hacia la investigación concreta. Pero, también, una especie de llamada de atención, tanto a un uso excesivamente subordinado al precepto de no intervención del moderador en los grupos de discusión, como a un uso que convierte las dinámicas en una especie de entrevista grupal, que no tiene en cuenta las intervenciones del moderador en el análisis más que en la trivial separación entre discurso espontáneo del grupo y discurso no espontáneo. M ientras que las primeras parecen partir del miedo a impregnar la dinámica de la subjetividad de la moderación, en todo momento y para todo objetivo, las otras simplemente no tienen en cuenta la subjetivización de los observados de tal subjetivización del observador.

Por supuesto, como paso desde la reflexividad teórica a la metodológica, se reconoce muy lejos de hacer camino. Un camino que abandona la rígida proposición de una autopista encarnada por perspectivas que van en un solo sentido. Un camino que conduce entre el reconocimiento de la fuerza inercial de los instrumentos y el de la propia fuerza del investigador, para usar los instrumentos según sus intereses y capacidades, pues, como señalan Grignon y Passeron: «C onviene saber qué es lo que pueden hacer los instrumentos para no exigirles un trabajo que trasciende sus posibilidades. Está claro, ya lo sé (pero prefiero no darlo por supuesto), que la fuerza metodológica de un instrumento no es sino aquella con la que puede contar epistemológicamente quien la utiliza. D ar la importancia que corresponde a esta fuerza de inercia del instrumento significa también, para el sociólogo, no fiarse demasiado de sus propias fuerzas» (Grignon y Passeron, 1992: 61).

\section{Bibliografía}

ALon SO, L.E. (1996). «El grupo de discusión en su práctica: memoria, intertextualidad y acción comunicativa». Revista Internacional de Sociología.

AlvIRA, F. (1983). «Perspectiva cualitativa-perspectiva cuantitativa en la metodología sociológica». Revista Española de Investigaciones Sociológi cas, 22, p. 53-76.

BAJTIN, M. (1981). «Le discours dans la vie et dans la poésie». En T O d O ROV, T. (ed.). M ikhail Bajtine. Le principe dialogique. París: Seuil.

- (1986). Problemas de la poética de D ostoievski. M éxico: Fondo de Cultura Económica. BECK, U. (1992). Risk Society. Londres: Sage.

BECK, U .; GIDDEN S, A.; LASH , S. (1994). Reflexive M odernization. Cambridge: Polity Press.

BELL, D. (1976). El advenimiento de la sociedad post-industrial. M adrid: Alianza.

BотT, Elizabeth (1971). Family and Social N etwork. N ueva York: Free Press (hay traducción en Taurus).

BouRdieu, P. (1988). La distinción. M adrid: Taurus.

- (1991). «ntroduction á la socioanalyse». Actes de la Recherche en Sciences Sociales, 90, diciembre, p. 3-5.

- (1992). Réponses París: Seuil.

- (1993). La misère du monde. París: Seuil. 
Callejo, J. (1995). «La construcción del consumidor global». Si stema, 126.

- (1996). «Fatalidad del mercado y culturas de la producción». Sociología del trabajo, 26.

Callejo, J.; Vallejos, A.F. (1994). «Enfoque dialógico del grupo de discusión: diálogo e investigación social». Comunicación presentada en el II Encuentro Interdisciplinario de Retórica. Cádiz, 7 de diciembre de 1994.

CLIFFORD, J. (1995). Dilemas de la cultura. Barcelona: Gedisa.

CLIFFORD, J .; M ARCUS, G.E. (1991). Retóricas de la antropología. Gijón: Júcar.

Colem An, J.S. (1958). «Relational Analysis: The Study of Social O rganizations with Survey methods». H uman O rganization, 17(4), p. 28-36.

CONDE, F. (1987). «U na propuesta de uso conjunto de las técnicas cuantitativas y cualitativas en la investigación social. El isomorfismo de las dimensiones topológicas de ambas técnicas». Revista Española de Investi gaci ones Sociológicas, 39, p. 213-224.

- (1990). «Un ensayo de articulación de las perspectivas cuantitativa y cualitativa en la investigación social». Revista Española de Investigaciones Sociológicas, 51, p. 91-120.

- (1996). La vivienda en Huelva. Sevilla: Junta de Andalucía.

García Selgas, F. (1994). Teoría social y metateoría hoy. M adrid: CIS.

GeERTZ, C. y otros (1991). El surgimiento de la antropología postmoderna. M éxico: Gedisa.

GIDDEN S, A. (1984). The Constitution of Society. Londres: Polity Press.

- (1995a). M odernidad e identidad del yo. M adrid: Península.

- (1995b). La transformación de la intimidad. Sexualidad, amor y erotismo en las socie dades modernas. M adrid: C átedra.

- (1995c). «Foucault à propos de la sexualité». Soci etés, 48, p. 107-120.

G offm AN, E. (1972). Strategic interaction. Filadelfia: U niversity Pennsylvania Press.

- (1987). La presentación del yo en la vida cotidiana. M adrid: Amorrortu-M urguia. Go uld ner, A. (1970). La crisis de la sociología occidental. Buenos Aires: Amorrortu. Gran ovetTeR, M . (1973). «T he Strenght of Weak Ties». American Journal of Sociology, 78, p. $1.360-1.380$.

- (1976). «N etwork Sampling: Some First Steps». Amercian Journal of Sociology, 81, p. 1.267-1.303.

GrATH OFF, R. (1990). The structure of social inconsistences. La H aya: M artin Nijhoff. Grign on , C.; PASSERO N , J.C. (1992). Lo culto y lo popular. M adrid: La Piqueta.

GuBA, E.G. (1990). The paradigm dialog. Londres: Sage.

IBÁÑ̃EZ, J. (1979). M ás allá de la sociología. M adrid: Siglo XXI.

- (1985). Del algoritmo al sujeto. M adrid: Siglo XXI.

- (1990). «Perspectivas de la investigación social». En García Ferran do, M .; ALVIRA, F.; I BÁÑ EZ, J. (comps.). El análisis de la realidad social. M adrid: Alianza.

- (1994). El regreso del sujeto. M adrid: Siglo XXI.

JAM ESON, F. (1991). Postmodernity, or the cultural logico of late capitalism. Durham: Duke U niversity Press.

JANIS, I.L. (1974). «dentificación de grupo en condiciones de peligro externo». En CARTWRIGHT, D .; ZAN DER, A. Dinámica de grupos Investigación y teoría. M éxico: Trillas.

LAM O DE ESPIN OSA, E. (1990). La sociedad reflexiva: sujeto y objeto del conocimiento sociológico. M adrid: CIS. 
- (1993). «La interacción reflexiva». En LAM O DE EsPIN OSA, E.; RodrígUEZ-IBÁÑEZ, J.E. (comps.). Problemas de teoría social contemporánea. M adrid: CIS.

- (1994). «La sociedad reflexiva. Ciencia social y sociedad del conocimiento». En LAM O dE ESPIN OSA, E.; GonZÁlez, J.M.; T ORRES, Cristóbal. La sociología del conocimiento y de la ciencia. M adrid: Alianza.

LAPASSADE, G. (1971). L'arpenteur. París: Epi.

LOUREAU, R. (1970). L'analyse institutionnelle. París: M inuit.

- (1977). «ntroducción». En VV.AA. El análisis institucional. M adrid: Campo Abierto.

LozARES, Carlos (1996). «La teoría de redes sociales». Papers, 48.

LYON, D . (1996). Postmodernidad. M adrid: Alianza.

MERTON, R.K. (1972). Teoría y estructuras sociales. M éxico: Fondo de Cultura Económica.

- (1980). Ambivalencia sociológica y otros ensayos. M adrid: Espasa Calpe.

N OYA, F.J . (1994). «M etodología, contexto y reflexividad. U na perspectiva constructivista y contextual ista sobre la relación cualitativo-cuantitativo en la investigación social ». En D ELGADO , J.M .; G UTIÉRREZ, J. M étodos y técnicas cual itativas de investigación en ciencias sociales. M adrid: Síntesis.

O LM STED, M .S. (1992). El pequeño grupo. M éxico: Paidós.

Rodríguez, J.A. (1995). Análisis estructural y de redes M adrid: C entro de Investigaciones Sociológicas.

RoSALD O, R. (1993). Culture and Truth. The remaking of Social Analysis. Londres: Routledge.

RuBY, J. (1991). A crack in the mirror: reflexive perspectives in anthropology. Filadelfia: U niviversity of Pennsylvannia Press.

SAN D YWELL, B. (1996). Reflexivity and the crisis of Western Reason. Londres y N ueva York: Routledge.

SCHACHTER, S. (1974). «D esviación, rechazo y comunicación». En CARTWRIGHT, D .;

ZANDER, A. Dinámica de grupos. Investigación y teoría. M éxico: Trillas.

SCHECHNER, R. (1991). «Restorarion of Behabior». En RUBY, J. A crack in the mirror: reflexive perspectives in anthropology. Filadelfia: University of Pennsylvannia Press. ScH AW, M . (1980). D inámica de grupos. Barcelona: H erder.

SPREEN, M arinus (1994). "Social network analysis and addiction research».

Comunicación presentada en la International Conferencé Alcohol D rugs H IV/AID S and Gambling: N ew international experiences compared. Rotterdam.

SPREen, M arinus; Z WAAGSTRA, Ronald (1994). «Personal network sampling, outdegree analysis and multilevel analysis: introducing the network concept in studies of hidden populations». International Sociology, vol. 9, núm. 4, p. 475-491.

Su d M AN, Seymur; Kalto N, Graham (1986). «N ew D evelopments in the sampling of special populations». Annual Review of Sociology, 12, p. 401-29.

SteleR, F. (1991). Research and reflexivity. Londres: Sage.

Vallejos, A.F. (1995). «Perspectiva dialógica del grupo de discusión». Comunicación

presentada al V C ongreso Español de Sociología. Granada, 29 de septiembre de 1995.

W ATTERS, John K.; BIERN ACKI, Patrick (1989). «Targeted Sampling: O ptions for the

Study of H idden Populations». Social Problems, vol. 34, p. 416-430.

W ATZLAWICK, Paul; KRIEG, Peter (1994). El ojo del observador. Barcelona: Gedisa.

W RIGHT M ILLS, C. (1993). La imaginación sociológica. M adrid: Fondo de Cultura Económica. 